\title{
Carbon starvation of Pseudomonas aeruginosa biofilms selects for dispersal insensitive mutants
}

Harikrishnan A. S. Nair ${ }^{1,2,3+}$, Sujatha Subramoni ${ }^{1+}$, Wee Han Poh ${ }^{1}$, Nabilah Taqiah Binte Hasnuddin', Martin Tay ${ }^{1,4}$, Michael Givskov ${ }^{1,5}$, Tim Tolker-Nielsen ${ }^{5}$, Staffan Kjelleberg ${ }^{1,6}$, Diane McDougald ${ }^{1,7^{*}}$ (I) and Scott A. Rice ${ }^{1,6,7^{*}}$

\begin{abstract}
Background: Biofilms disperse in response to specific environmental cues, such as reduced oxygen concentration, changes in nutrient concentration and exposure to nitric oxide. Interestingly, biofilms do not completely disperse under these conditions, which is generally attributed to physiological heterogeneity of the biofilm. However, our results suggest that genetic heterogeneity also plays an important role in the non-dispersing population of P. aeruginosa in biofilms after nutrient starvation.

Results: In this study, $12.2 \%$ of the biofilm failed to disperse after $4 \mathrm{~d}$ of continuous starvation-induced dispersal. Cells were recovered from the dispersal phase as well as the remaining biofilm. For $96 \mathrm{~h}$ starved biofilms, rugose small colony variants (RSCV) were found to be present in the biofilm, but were not observed in the dispersal effluent. In contrast, wild type and small colony variants (SCV) were found in high numbers in the dispersal phase. Genome sequencing of these variants showed that most had single nucleotide mutations in genes associated with biofilm formation, e.g. in wspF, pilT, fha1 and aguR. Complementation of those mutations restored starvation-induced dispersal from the biofilms. Because c-di-GMP is linked to biofilm formation and dispersal, we introduced a c-di-GMP reporter into the wild-type P. aeruginosa and monitored green fluorescent protein (GFP) expression before and after starvationinduced dispersal. Post dispersal, the microcolonies were smaller and significantly brighter in GFP intensity, suggesting the relative concentration of c-di-GMP per cell within the microcolonies was also increased. Furthermore, only the RSCV showed increased c-di-GMP, while wild type and SCV were no different from the parental strain.
\end{abstract}

Conclusions: This suggests that while starvation can induce dispersal from the biofilm, it also results in strong selection for mutants that overproduce c-di-GMP and that fail to disperse in response to the dispersal cue, starvation.

Keywords: Biofilm development, C-di-GMP, Bioreporter, Pseudomonas aeruginosa, Image-based quantification, Starvation, Morphotypic variants, Dispersal

*Correspondence: Diane.McDougald@uts.edu.au; rscott@ntu.edu.sg ${ }^{\dagger}$ Harikrishnan A. S. Nair and Sujatha Subramoni contributed equally to this work.

${ }^{7}$ The Ithree Institute, University of Technology Sydney, Sydney, Australia Full list of author information is available at the end of the article

\begin{abstract}
Background
Upon maturation, biofilm microcolonies release cells in an active process called dispersal, which results in dispersal cells colonising new niches [1]. Bacteria typically disperse from biofilms in response to environmental cues, such as nutrient starvation, oxygen limitation and some signal molecules, e.g. nitric oxide (NO) [2-4]. Biofilm dispersal is an active, energy requiring process that is regulated by multiple molecular pathways $[5,6]$. Studies
\end{abstract}


of biofilm dispersal are of broad interest, especially where manipulation of the dispersal response can be exploited to control biofilms in clinical and industrial settings. For example, NO releasing hydrophobic polymers are known to reduce adhesion of bacteria to indwelling medical devices such as catheters and arteriovenous grafts [7]. While it is clear that these cues or pathways can be manipulated for biofilm control, e.g. by exposing biofilms to exogenously added NO donors, exposure to dispersing conditions rarely results in complete biofilm dispersal $[8,9]$. Fluctuations in nutrient concentrations (increases or decreases), also trigger biofilm dispersal, although, as observed for NO, dispersal is not complete and some biofilm remains $[3,10,11]$. A monospecies biofilm growing on a single carbon substrate will develop zones of differing oxygen or nutrient availability, e.g. oxygenic, limited oxygenic and anoxic zones, due to incomplete diffusion of oxygen from the medium or as a consequence of rapid utilisation by the bacteria within the biofilm [12-14]. Thus, a facultative aerobic bacterial biofilm may grow aerobically in the oxygenic zone and by fermentation or oxygen-independent respiration in the anoxic zone [12, 15]. Hence, dispersal as a consequence of these environmental cues reflects cellular physiology and it is likely that incomplete dispersal is a reflection of physiological heterogeneity across the biofilm [16].

We have previously shown that biofilms of $P$. aeruginosa disperse upon carbon source starvation and that starvation results in a rapid reduction in c-di-GMP [4].
We further showed that dispersal induced as a result of carbon source starvation was an active, energy requiring process [10]. While carbon source limitation resulted in dispersal, approximately $40 \%$ of the biofilm biomass remained, even after $24 \mathrm{~h}$ of starvation. It is also frequently observed that mature biofilms produce morphotypic variants, in some cases up to $60 \%$ of the recovered isolates and that the appearance of these variants is associated with the dispersal phase of biofilm development [17-21]. Such morphotypic variants are often better at forming biofilms than the parental strain and such variants can outcompete the parental strains when co-cultured [22]. Here, we investigated biofilms of $P$. aeruginosa after starvation-induced dispersal and show that the nondispersing biomass has a high proportion of morphotypic variants and that these variants have SNPs in genes associated with biofilm formation, in particular, genes associated with the production or degradation of c-di-GMP.

\section{Results}

Biofilm dispersal due to starvation is incomplete

$P$. aeruginosa biofilms were formed in flow cells for $4 \mathrm{~d}$, after which time starvation was induced by replacing the medium with glucose-free M9. The biovolume was calculated using image-based quantification after staining for live and dead cell populations. Biovolumes of live cells decreased from $4.55 \times 10^{6}$ to $1.18 \times 10^{6} \mu \mathrm{m}^{3} \mu \mathrm{m}^{-2}$ within $24 \mathrm{~h}$ of the start of starvation, indicating significant dispersal in response to starvation (Fig. 1). The biomass of

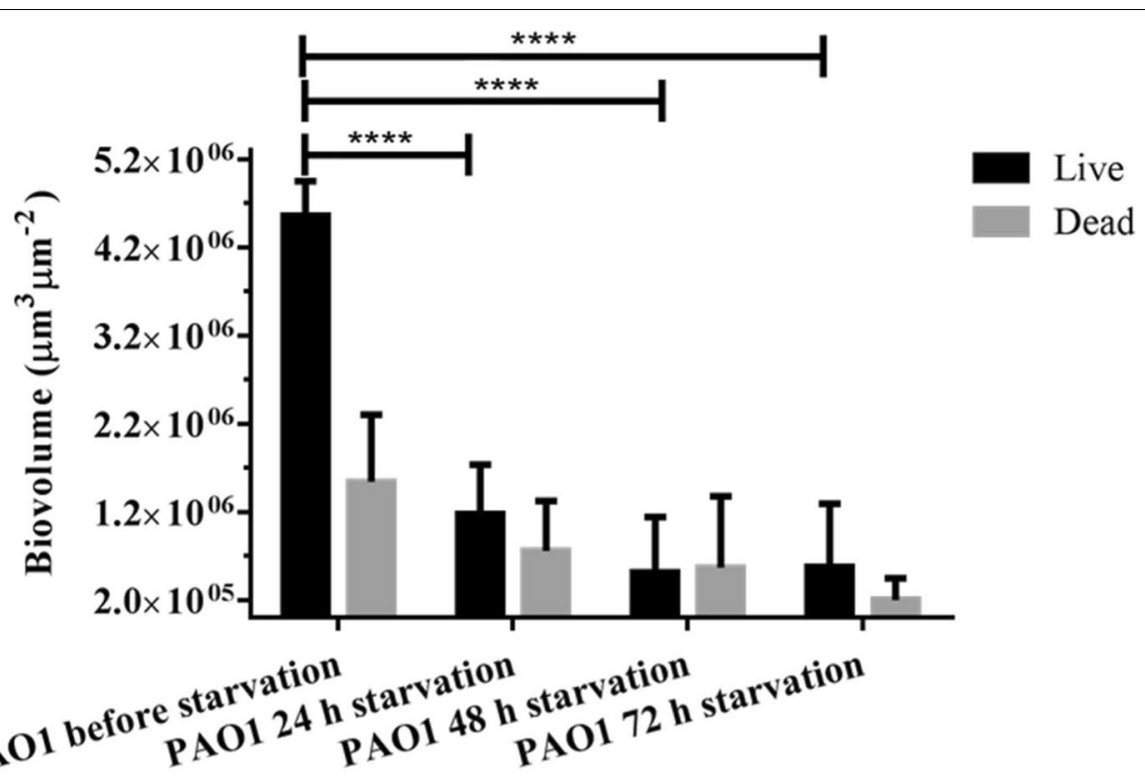

Fig. 1 Characterisation of P. aeruginosa biofilms during starvation. The biofilm was stained for viability with the BacLight ${ }^{\mathrm{TM}}$ Live-Dead $^{\circledR}$ reagents, and the biovolume was calculated as average of 3 independent experiments from CLSM images using the Imaris software package. For each experiment 15 CLSM images were taken. Error bars represent standard deviations $(n=3)$. Asterisks indicate values were significantly different between samples (Two-way ANOVA followed by Sidak's post-test; ${ }^{* * *}<\mathrm{P} 0.0001$ ) 
dead cells decreased from $1.4 \times 10^{6}$ to $2.6 \times 10^{5} \mu^{3} \mu \mathrm{m}^{-2}$ after $24 \mathrm{~h}$ of starvation. However, after $48 \mathrm{~h}$ and $72 \mathrm{~h}$ of starvation, the remaining live cell biofilm biovolume was approximately $5.13 \times 10^{5}{\mu \mathrm{m}^{3}}^{3} \mathrm{~m}^{-2}$ and $5.57 \times 10^{5}$ $\mu \mathrm{m}^{3} \mu \mathrm{m}^{-2}$, respectively. The biomass of dead cells was reduced to $2 \times 10^{5}$ at the end of $72 \mathrm{~h}$ of starvation. Thus, although there was substantial dispersal upon starvation, much of the biomass (12.2\%) remained attached in the flow cell (Fig. 1). In contrast, we have shown previously that biovolume per unit area of $P$. aeruginosa biofilms increases steadily until day 4 and remains constant until day 7 in the absence of starvation [23]. Therefore, for biofilms under natural nutrient conditions there is no appreciable dispersal until day 7.

\section{Characterisation of morphotypic variants isolated from starved biofilms}

When biofilms containing the pCdrA::gfp (ASV) reporter were imaged after $24 \mathrm{~h}$ of starvation, it was observed that the remaining biomass was strongly fluorescent, suggesting that those microcolonies had very high intracellular c-di-GMP levels (Fig. 2). It has been previously shown that biofilm dispersal is associated with the formation of morphotypic variants $[18,24]$ and such variants often are mutants that overproduce c-di-GMP $[25,26]$.

The biofilm cells formed three distinct morphotypes, one that was similar to the WT P. aeruginosa, small colony variants (SCVs) and rugose small colony variants (RSCVs). Therefore, biofilms were collected after $4 \mathrm{~d}$ of starvation and cells were serially diluted and plated onto agar plates. The cell numbers were stable at $48 \mathrm{~h}$ and $72 \mathrm{~h}$ in our earlier experiment; therefore we wanted to check a longer time point and hence chose $96 \mathrm{~h}$ over $72 \mathrm{~h}$ for enumeration. Prior to starvation, the effluent had high numbers of WT P. aeruginosa $\left(3.2 \times 10^{7} \mathrm{cfu} \mathrm{m}^{-1}\right)$ (Fig. 3) and a small number $\left(1.9 \times 10^{6} \mathrm{cfuml}^{-1}\right)$ of SCVs. After $1 \mathrm{~h}$ of starvation, the effluent contained $1.2 \times 10^{7} \mathrm{cfu} \mathrm{ml}^{-1}$ wild type and $1.6 \times 10^{6} \mathrm{cfu} \mathrm{ml}^{-1} \mathrm{SCV}$. A similar trend was observed after $24 \mathrm{~h}, 48 \mathrm{~h}$ and $96 \mathrm{~h}$ of starvation, where the effluent always contained a higher number of WT $P$. aeruginosa and relatively fewer SCVs. For each time-point separate flow cells were cultivated, the flow cell was disconnected and flushed with medium to extract biomass. The biofilm biomass obtained after $1 \mathrm{~h}, 24 \mathrm{~h}, 48 \mathrm{~h}$ and $96 \mathrm{~h}$ of starvation showed a similar trend of higher levels of WT P. aeruginosa and lower levels of SCVs. Only the biofilm biomass extracted after $96 \mathrm{~h}$ starvation was found to contain RSCVs. At this time point the numbers of bacteria in the extracted biomass included $4 \times 10^{5} \mathrm{cfu} \mathrm{ml}^{-1}$ RSCVs, $6 \times 10^{5} \mathrm{cfu} \mathrm{ml}^{-1} \mathrm{SCVs}$ and $1.3 \times 10^{7} \mathrm{cfu} \mathrm{ml}^{-1} \mathrm{WT}$ bacteria. The lower numbers of WT bacteria enumerated at $96 \mathrm{~h}$ enabled detection of RSCVs in the biofilm biomass at this timepoint. Control biofilms without starvation were also tested and the number of variants were enumerated from both effluent and biofilm biomass for comparison as shown (Fig. 3).

Five representatives of each morphotype were genome sequenced. The WT isolates (WT1-5) harboured one or more mutations (Table 1). For example, the WT1 had a deletion in $f l i H$ and a substitution in mexT. WT2 had only one mutation, an insertional mutation in PA4321, a hypothetical gene. WT3 and WT5 had several SNPs in pilT, which is known to be important for twitching motility $[27,28]$. WT4 had an insertional mutation in fha1 coding for a component of the type VI secretion system [29]. SCV1 and SCV2 had mutations in wspF, coding for

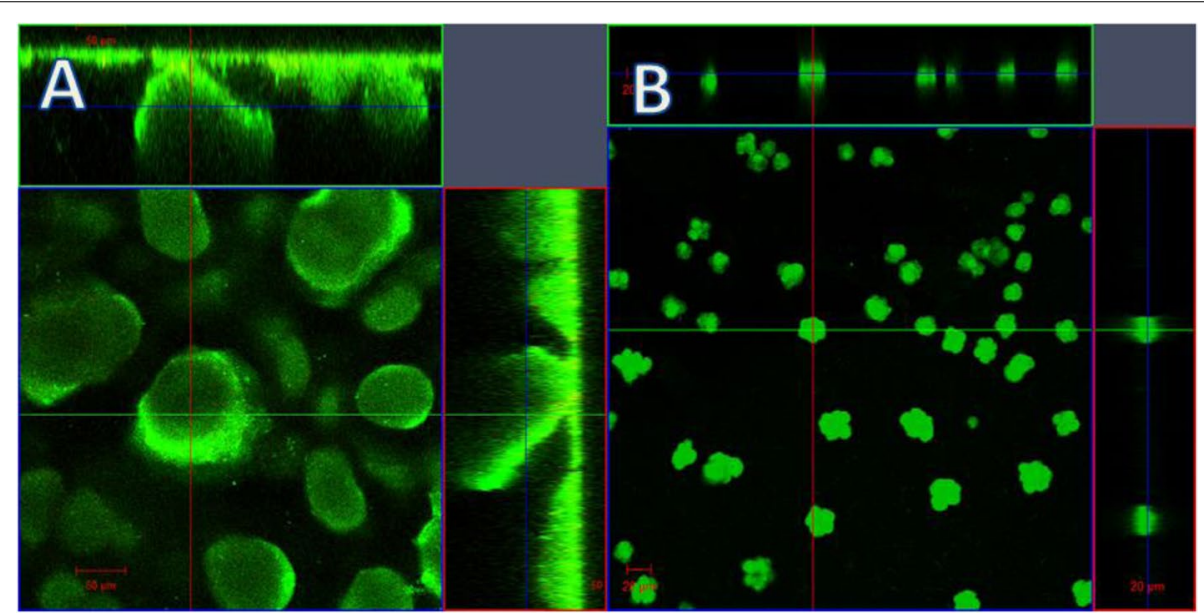

Fig. 2 Orthogonal view of P. aeruginosa microcolonies before and after starvation. A) biofilm before starvation B) colonies after $72 \mathrm{~h}$ of starvation. P. aeruginosa contained the c-di-GMP responsive reporter construct as described [39]. Orthogonal view showing top panel $x-z$ plane, right $y-z$ and middle corresponding x-y plane. Magnification 20x. Scalebar: $50 \mu \mathrm{m}$ (A) or $20 \mu \mathrm{m}$ (B) 

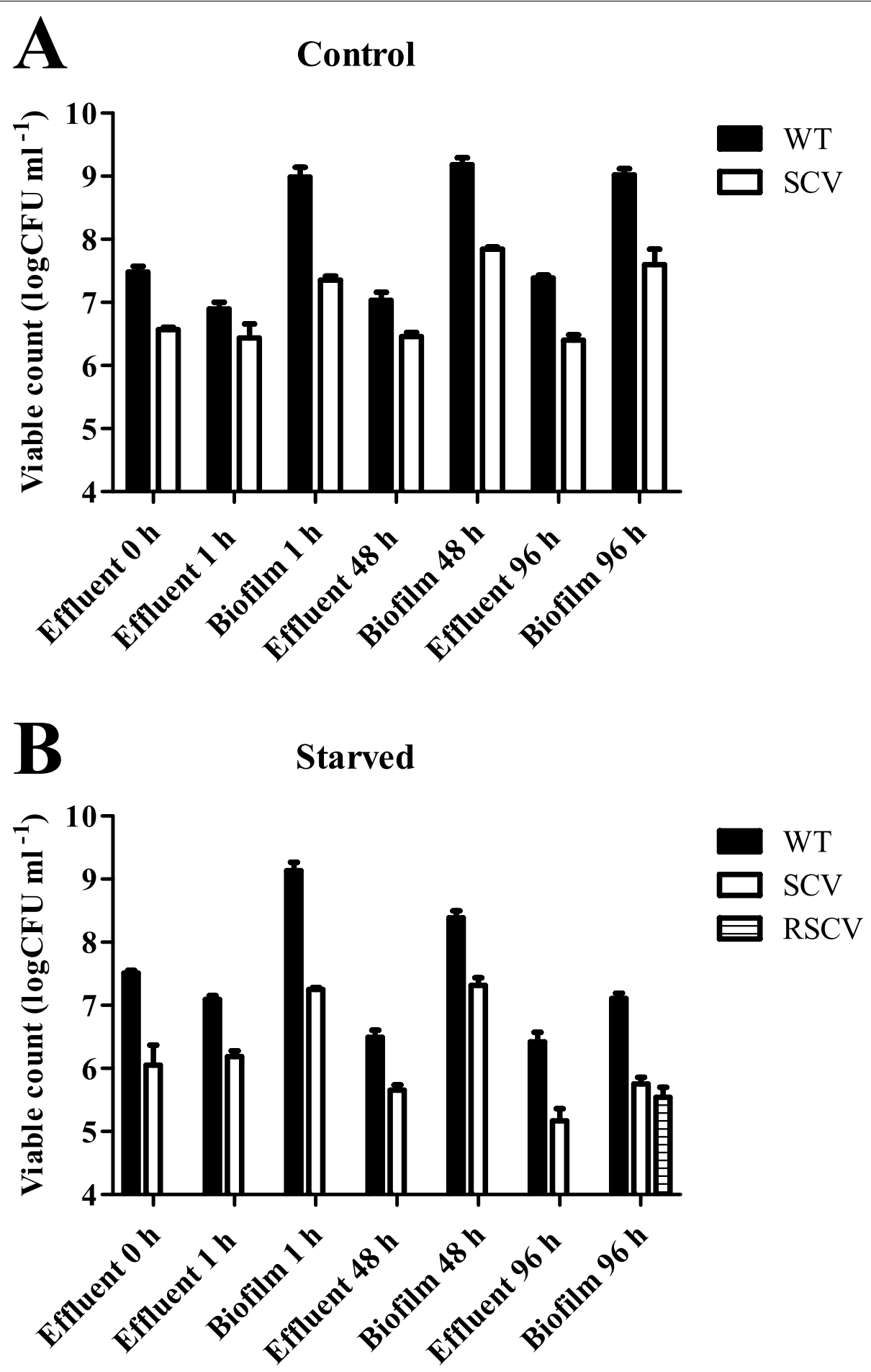

Fig. 3 Colony variants of P. aeruginosa during biofilm starvation. Effluent from biofilms pre- and post- starvation was serially diluted and plated onto LB agar for enumeration. For each time point, the biomass was also harvested from the flow cells, serially diluted and plated onto LB agar to ennumerate morphotypic variants. Independent flow-cells were run for each time point for control (A) and starved (B) conditions 
Table 1 Identification of genetic differences between the $P$. aeruginosa wild type and non-dispersing isolates

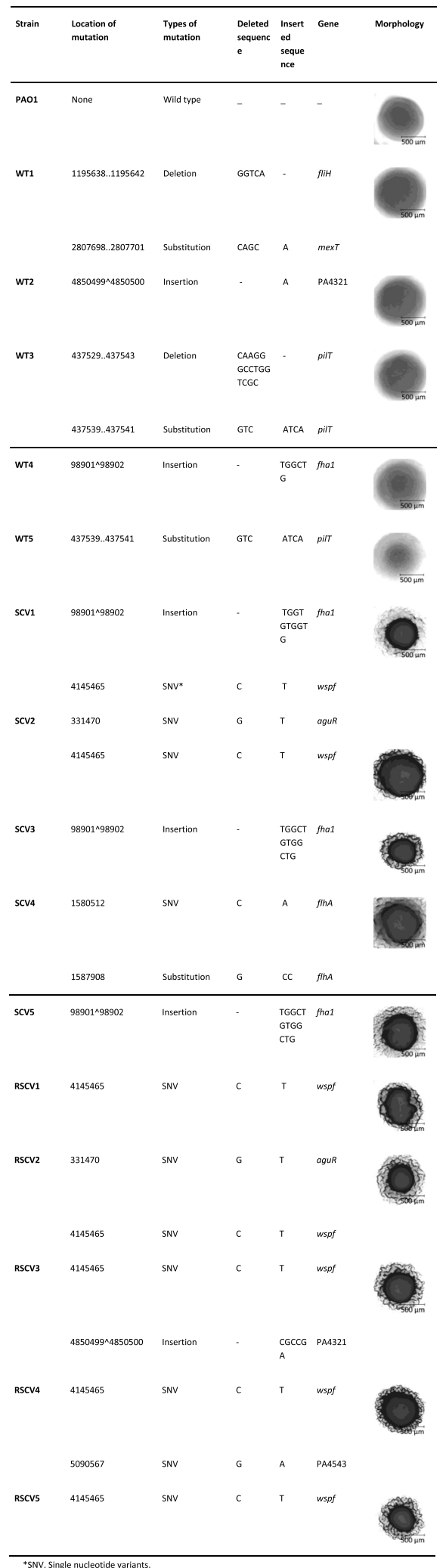

a methylesterase protein of the chemosensory signaltransduction system [30,31]. Loss of WspF function leads to over expression of the diguanylate cyclase WspR and hence, increased biofilm formation [30]. Some of these morphotypic variants contained additional mutations, including fha1 in SCV1 and SCV3 and $f l h A$, involved in flagella biosynthesis in SCV3, SCV4 and SCV5 [32]. All of the RSCVs contained a mutation in $w s p F$ and this mutation was consistent with their rough colony morphotype as described previously $[22,33]$. Additional mutations in the RSCVs include aguR in RSCV2, mutation of PA4321 along with wspF in RSCV3 and mutation of PA4543 in RSCV4. RSCV5 and RSCV1 were genotypically the same, containing a single nucleotide variant (SNV) at the same position in the $w s p F$ gene.

To further characterise the morphotypic variants isolated from starved biofilms, the isolates were tested for c-di-GMP production, biofilm formation and dispersal in response to starvation. All colonies with a WT phenotype had pCdrA:: gfp expression levels $(559 \pm 11,553 \pm 80$, $525 \pm 25 \mathrm{RFU})$, that were similar to the parental strain (555 \pm 42 RFU), indicating they have similar concentrations of c-di- GMP (Fig. 4). SCVs had slightly higher c-di-GMP levels $(734 \pm 37,682 \pm 28,567 \pm 58$ RFU) compared to WT. All of the RSCVs had considerably higher c-di-GMP levels compared to WT, although RSCV2 $(1289 \pm 134$ RFU) had only half of the c-di-GMP levels of RSCV1 (2367 \pm 152 RFU) and RSCV3 (1871 \pm 324 RFU). The RSCVs were also found to form pellicles at the airliquid interface of planktonic cultures (data not shown), a phenotype characteristic of the high c-di- GMP mutant wspF [30].

When these biofilm isolates were tested for their response to starvation induced dispersal, it was observed that the WT-like isolate, WT1, responded similarly to the parental WT strain (Fig. 5). WT1 showed 56.8\% dispersal in the first $24 \mathrm{~h}$ post induction of starvation and showed no change in dispersal at 48 or $96 \mathrm{~h}$. In contrast, none of the SCV or RSCV variants tested showed any significant dispersal upon starvation, even after $96 \mathrm{~h}$.

\section{Complementation restores starvation induced dispersal phenotype to RSCV biofilm isolate}

To confirm the effect of the mutations found in the SCV and RSCV isolates were responsible for their failure to disperse upon carbon starvation, we introduced wildtype copies of the genes affected. To carry out complementation analyses, RSCV1, WT5 and SCV5 mutants carrying plasmids with the respective wild type copies of $w s p F$, pilT and fha 1 were generated. These three strains were selected because they had only a single gene that was mutated relative to the parental strain. All three colony morphotype variants, RSCV1, WT5 


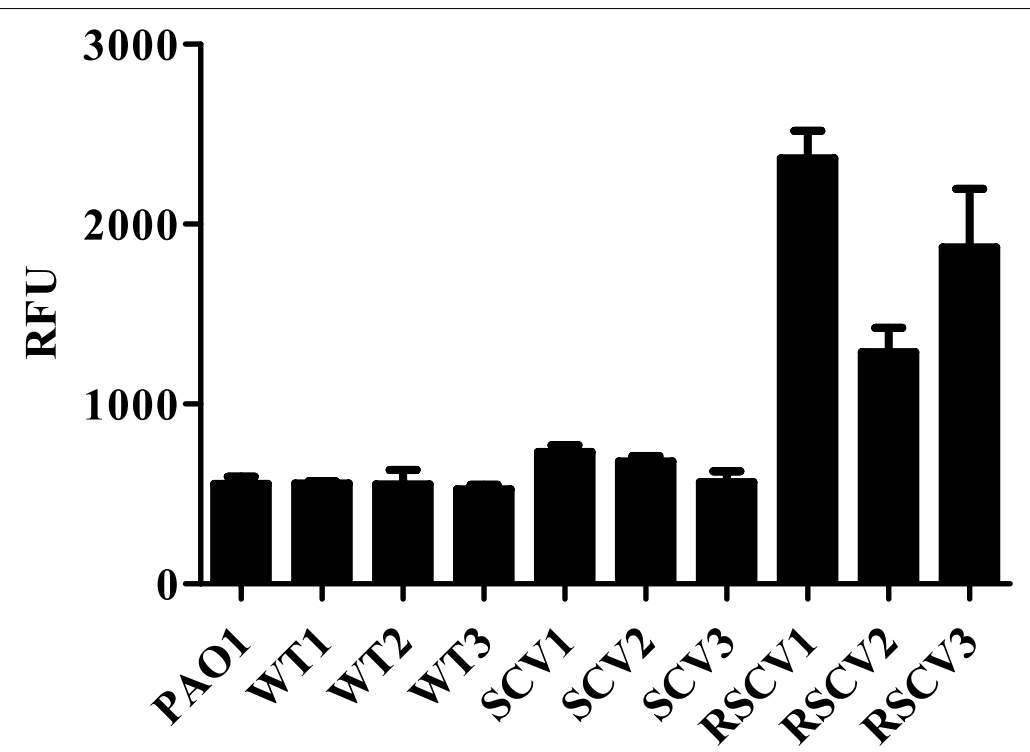

Fig. 4 C-di-GMP levels of colonies that fail to disperse upon starvation measured using the cdrA.::gfp reporter. Individual isolates were grown in M9 glucose medium overnight and $2 \mathrm{ml}$ of culture was transferred to a 24 well plate in triplicate. GFP fluorescence and OD were measured using a Tecan plate reader. RFUs were calculated as GFP/OD 600 . Each data point is the average of three technical replicates

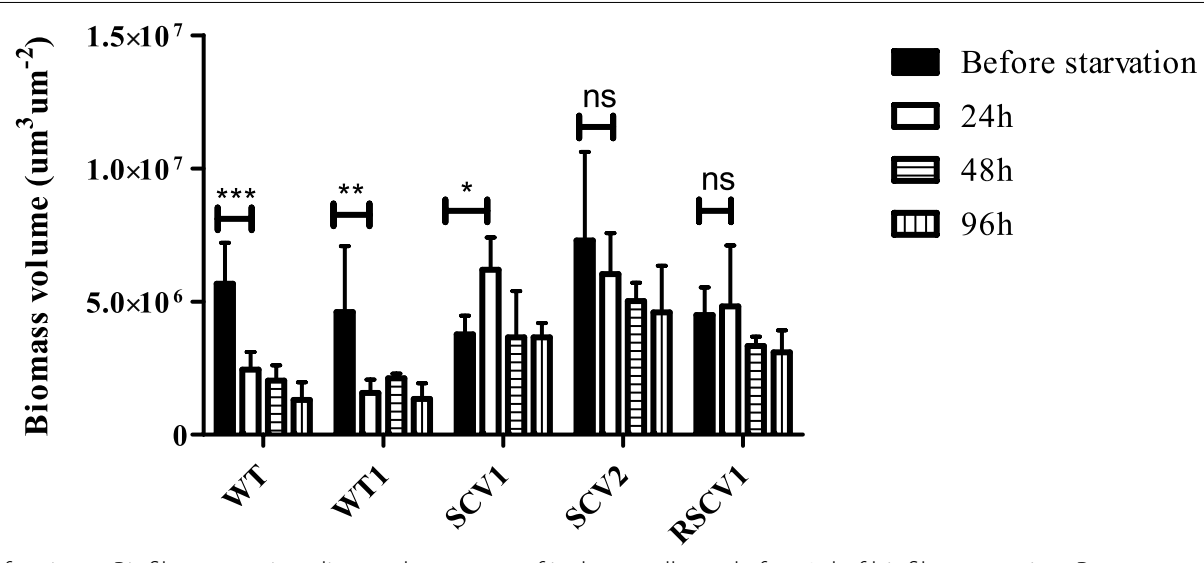

Fig. 5 Starvation of variants. Biofilm starvation dispersal response of isolates collected after $4 \mathrm{~d}$ of biofilm starvation. Representative morphotypic isolates were grown in flow cells and starvation was induced at day 4. Biomass biovolumes were calculated as average of 3 independent experiments by CLSM images using IMARIS software. For each experiment 5 CLSM images were taken. Error bars represent standard deviations $(n=3)$. Asterisks indicate that the values are significantly different (Two-way ANOVA followed by Tukey's post-test; $\left.{ }^{* * *}<\mathrm{P} 0.001,{ }^{* *}<\mathrm{P} 0.01,{ }^{*}<\mathrm{P} 0.05\right)$

and SCV5 were restored to the wild type colony morphology upon complementation. Batch biofilms were cultivated with the mutants carrying either the empty vector (pBBR) or complementing clones (pBBRwspF, pBBRpilT or pBBRfha1), subjected to starvation conditions and dispersal was monitored. Analysis of dispersal phenotypes showed that only the RSCV1 mutant was complemented for this phenotype when WT wspF was provided in trans (Fig. 6). WT P. aeruginosa had a dispersal rate of $0.57 \mathrm{~min}^{-1}$ whereas RSCV1 mutant and RSCV1/pBBR had dispersal rates of $0.02 \mathrm{~min}^{-1}$ and $0.12 \mathrm{~min}^{-1}$, respectively. The RSCV1 mutant carrying $\mathrm{pBBR} w s p F$ was restored to almost WT levels of dispersion rates at $0.46 \mathrm{~min}^{-1}$ suggesting that $\mathrm{WspF}$ is required for dispersal during starvation.

\section{Discussion}

Many studies have shown that c-di-GMP plays a key role in regulating the switch between bacterial life styles, from free-living planktonic bacteria to sessile biofilms. Elevated levels of c-di-GMP are commonly associated with biofilm growth, and reductions in this 


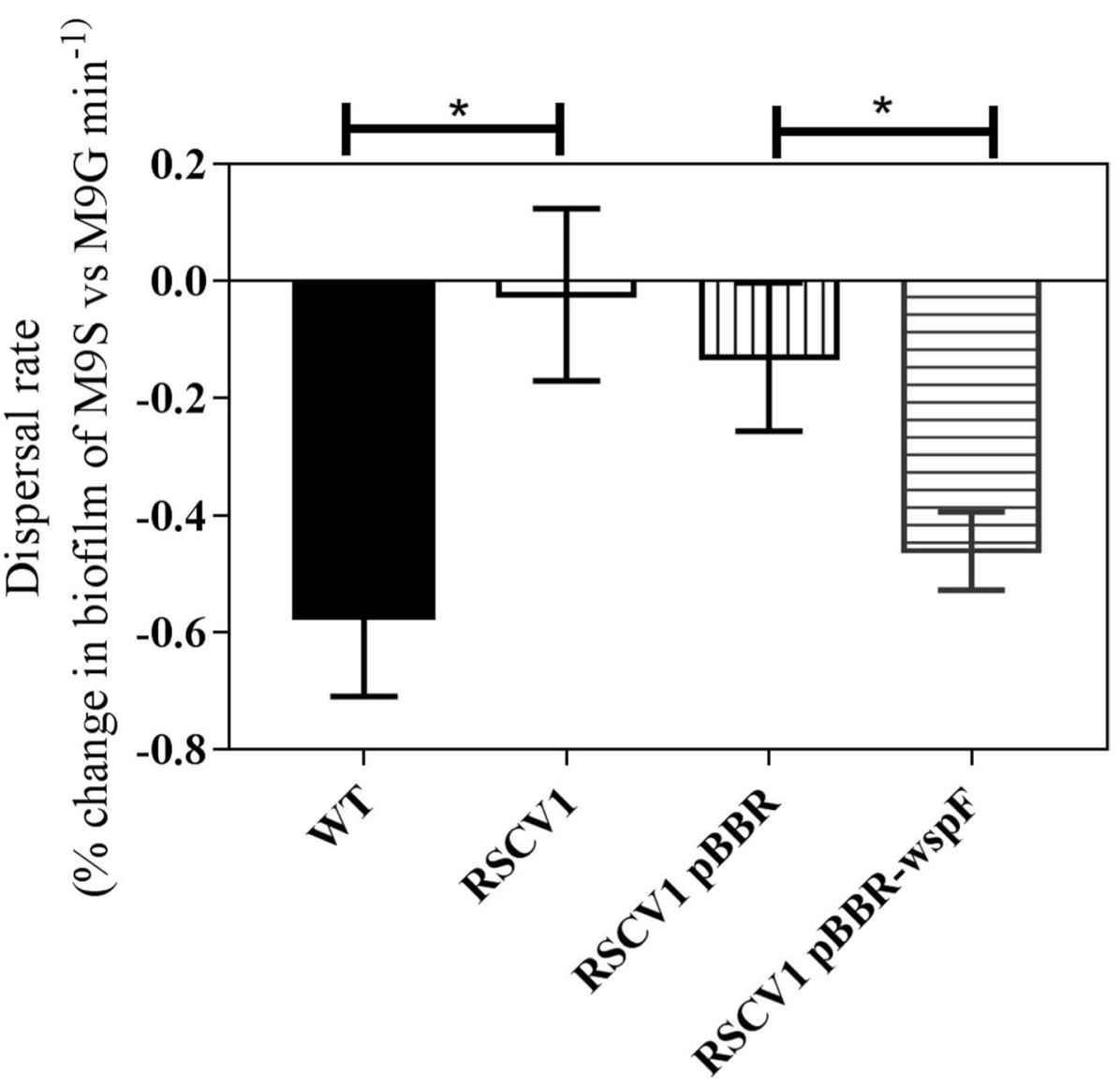

Fig. 6 Complementation of non-dispersal phenotype of RSCV1 mutant under starvation conditions. Batch biofilms of WT, RSCV1, RSCV1/pBBR and RSCV1/pBBRwspF were cultivated for $6 \mathrm{~h}$ and starved. Biofilm biomass was quantified by crystal violet staining at different time points. Percentage biofilm changes were plotted against time and the rate of change of biofilm reduction was obtained through linear regression. Data shown are average values \pm SD from 3 independent experiments. M9G-M9 medium containing glucose; M9S-M9 salts without carbon source. Asterisks indicate that the values are significantly different between strains (One-way ANOVA followed by Tukey's post-test; ${ }^{*}<\mathrm{P} 0.05$ )

second messenger can result in the dispersal of biofilms [34]. Dispersal cues responsible for altering the intracellular concentrations of c-di-GMP include oxygen limitation, cues such as $\mathrm{NO}$ and nutrient starvation [4, $9,10,15]$. While starvation and NO can induce dispersal, it is frequently observed that dispersal is incomplete $[3,11]$. It was recently demonstrated that the non-dispersing cells have increased levels of flavohemoglobin (Fhp) that scavenge and inactivate NO, thus preventing further dispersal [35]. Thus, it was of interest to investigate the effect of starvation as a dispersal cue in $P$. aeruginosa to assess the non-dispersing population.

Even though starvation did induce dispersal, 12.2\% of the initial biomass remained in the flow cell after $3 \mathrm{~d}$ of continuous starvation (Fig. 1). Isolation and genetic characterisation of these dispersal insensitive colonies showed that they contained mutations. Since the WT morphotype colonies show dispersal similar to the parent strain, it is likely that these mutants would account for a smaller percentage of dispersal resistant mutants when compared to RSCV and SCV. The presence of morphological variants in the biofilm is a common observation $[18,24,36]$ and morphotypic variants such as SCVs and RSCVs have been correlated with the persistence of $P$. aeruginosa during chronic infection of cystic fibrosis patients [33]. It is likely that these morphotypic variants arise in the population through natural mutational processes and that some of these natural mutants, in particular SCVs and RSCVs, were selected for in the dispersal resistant biomass. The isolates with WT morphotype harboured mutations in the $\mathrm{fliH}$ and mexT genes; however, their response to starvation and their intracellular c-di-GMP concentrations were similar to the parent strain. The $f l i H$ gene encodes a protein important for flagella biosynthesis [37] and a mutation would render $P$. aeruginosa nonmotile. The gene mexT encodes is a LysR-family regulator that modulates multi-drug efflux systems, resulting in increased 
resistance to diverse antibiotics [38]. Complementation of the mutants with $w s p F$ restored WT dispersal, while complementation with pilT or fha1 did not. As for the WT isolates, none of the SCVs showed elevated levels of c-di-GMP. In contrast, all of the RSCVs contained $w s p F$ mutations and showed elevated levels of c-di-GMP, as shown using the pCdrA::gfp monitor (Fig. 6), although the concentration of c-di-GMP differed between isolates, possibly due to secondary mutations (e.g. aguR, PA4321, PA4543) [16]. Therefore, at least for these strains, the failure to disperse may be a consequence of being locked into the biofilm mode of growth. This is supported by the observation that the RSCV morphotypes do not appear in the biofilm effluent, but are strongly enriched in the non-dispersing biomass, even after $96 \mathrm{~h}$ of starvation.

These mutants most likely pre-exist in the biofilm and are strongly selected for under the conditions used here. It is likely that other triggers of biofilm dispersal, e.g. oxygen limitation or NO treatment may also select for high c-di-GMP producing mutants that are defective in dispersal. It would be of interest to determine if the same mutants arise that are impaired in dispersal, and would be the focus of future work which would include the specific signal response pathways for the different dispersal cues.

\section{Conclusions}

The results presented here show that, as for other dispersal cues such as NO, some cells in the biofilm population do not respond to the dispersal cue. In the case of NO, the lack of response was associated with induction of the NO scavenging enzyme, Fhp. In contrast, while starvation also failed to disperse the entire biofilm, we found that this was at least in part, due to the presence of a population of genetic variants that over produce c-di-GMP and hence may lock the cells into a biofilm phenotype. Thus, repeated cycles of dispersal and regrowth may represent a strong selection pressure that favours mutants in the population that are unable to disperse, further reinforcing biofilm growth.

\section{Methods}

\section{Bacterial strains and culture media}

P. aeruginosa PAO1 WT and SCV or RSCV mutant derivatives generated in this study are listed in Table 1. P. aeruginosa PAO1 mini-Tn7 CFP with a c-di-GMP reporter plasmid pCdrA::gfp (ASV) [39] was either cultured on Luria Bertani agar (LB broth, $10 \mathrm{~g}$ Tryptone, $10 \mathrm{~g} \mathrm{NaCL}$ and $5 \mathrm{~g}$ yeast extract / L, with $1.5 \%$ bacto agar) or M9 minimal medium ( $48 \mathrm{mM} \mathrm{Na} \mathrm{HPO}_{4} ; 22 \mathrm{mM}$ $\mathrm{KH}_{2} \mathrm{PO}_{4} ; 9 \mathrm{mM} \mathrm{NaCl} ; 19 \mathrm{mM} \mathrm{NH} \mathrm{NH}_{4} ; 2 \mathrm{mM} \mathrm{MgSO}$; $0.1 \mathrm{mM} \mathrm{CaCl}_{2}$ ) supplemented with $20 \mathrm{mM}$ glucose.

\section{Flow cell biofilms}

Biofilms were cultivated in three-channel flow cells (channel dimensions, $1 \times 4 \times 40 \mathrm{~mm}$ ) [40] and fed with M9 minimal medium supplemented with $20 \mathrm{mM}$ glucose as a carbon source at a flow rate of $9 \mathrm{mlh}^{-1}$. Each channel was inoculated with $0.5 \mathrm{ml}$ of diluted overnight culture containing approximately $1 \times 10^{8} \mathrm{cfu} \mathrm{ml}^{-1}[16]$.

\section{Confocal laser scanning microscopy and image analysis}

Biofilm images were acquired using a Carl Zeiss Confocal Laser Scanning Microscope CLSM 780 (Carl Zeiss, Germany) with a 20x objective (LD plan- Neofluar 20x / 0.4 Korr M27). Two separate optical channels were used to image the biofilm biomass and c-di-GMP reporter fluorescence. A stably-expressed cyan fluorescent protein (CFP), inserted into the genome, was used to quantify the biomass and GFP expression used to quantify c-diGMP [16]. Laser voltage was measured using a Sanwa ${ }^{\mathrm{TM}}$ $\mathrm{CD} 800 \mathrm{a}$ digital multimeter and kept at $0.587 \mathrm{~V}$ throughout the experiment. Pinholes of 50 and $58 \mu \mathrm{m}$ were used for CFP and GFP, respectively. An emission bandwidth of $440-503 \mathrm{~nm}$ was used for CFP and 497-598 $\mathrm{nm}$ was used for GFP. The master gain was set to 700 and the digital gain at 1.00 for acquisition of all of the images. Images were processed using ImageJ Version 1.47 [41].

\section{Starvation of planktonic and biofilm cultures}

Starvation of planktonic bacterial cultures was achieved by transferring cultures grown overnight into sterile $50 \mathrm{ml}$ Falcon tubes (Corning, Inc., U.S.) and centrifuging $(10,000 \mathrm{X} g)$ for $5 \mathrm{~min}$ at room temperature. Supernatants were discarded and bacterial cells were resuspended in M9 medium lacking a carbon source. The bacterial suspensions were then transferred to a new, sterile conical flask and incubated at room temperature with shaking $(180 \mathrm{rpm})$. To initiate starvation in flow cell biofilms, a separate media bottle containing M9 medium without a carbon source was connected to the pump and used as feed for the biofilm [16].

\section{Determination of colony morphology}

After $7 \mathrm{~d}$ of biofilm development, the flow cells were disconnected and bacterial cells were collected by flushing the biofilm several times with cold PBS using syringes connected to each end of the flow cell [42]. Biomass removal from the flow cells was validated by CLSM. The biomass was serially diluted with media (M9-glucose for normal growth, M9-without a carbon source for starvation) and plated onto LB agar plates. The plates were incubated for $3 \mathrm{~d}$ at room temperature and colonies were enumerated and morphology determined by use of a compound microscope (Zeiss Primo Star Compound Microscope) with $4 x$ magnification. 


\section{Variant analysis}

Planktonic cultures of isolated colony variants were grown overnight and DNA from each sample was extracted using QIAamp DNA Mini Kit (Qiagen, Netherlands) according to the manufacturer's protocol. The quantity of each DNA sample was determined using the Qubit DNA kit and the quality assessed using TapeStation. Libraries were prepared using the TruSeq DNA Sample Preparation Kit (Illumina, USA) and sequenced on the MiSeq (Illumina, USA) platform. The paired-end reads were trimmed to remove adapters and mapped to the $P$. aeruginosa reference genome (NCBI Reference Sequence: NC_002516.2) using CLC genomic workbench 6 (CLC bio, Denmark) [16].

\section{Complementation}

Sequences coding for wspF (1008bp), pilT (1035bp) and fha1 (1494bp) were amplified from the $P$. aeruginosa wild type (WT) by PCR and cloned into the KpnI-XbaI site of pBBRMCS-3. The resulting plasmids, pBBRwspF, pBBRpilT and pBBRfhal were introduced into RSCV1, WT5 and SCV5 respectively by electroporation. Colony morphology was assessed by compound microscopy (Zeiss) with $4 \mathrm{X}$ magnification. Batch biofilms were cultivated and dispersal assays carried out as follows; WT $P$. aeruginosa was grown overnight in LB supplemented with $50 \mu \mathrm{g} \mathrm{ml}^{-1}$ of tetracycline. Subsequently, overnight cultures were washed in $\mathrm{M} 9$ medium and resuspended to $\mathrm{OD}_{600}=1$. Twenty $\mu \mathrm{l}$ of the suspension was added to $1 \mathrm{ml}$ of fresh M9 medium supplemented with $0.4 \% \mathrm{w} / \mathrm{v}$ glucose in each well of a 24 well plate to a final $\mathrm{OD}_{600} \sim 0.02$. The 24 well plates were incubated for $6 \mathrm{~h}$ at $37^{\circ} \mathrm{C}$ with $180 \mathrm{rpm}$ shaking. At $6 \mathrm{~h}$, the medium was replaced with fresh M9 salts with or without $0.4 \% \mathrm{w} / \mathrm{v}$ glucose. To quantify biofilms, the medium was aspirated and each well washed once before staining with $0.1 \%$ crystal violet for $20 \mathrm{~min}$, at which time the crystal violet solution was removed and each well washed twice with $1 \mathrm{X}$ PBS. The remaining crystal violet was dissolved in ethanol and quantified using a microtitre plate reader (Tecan infinite pro M200). Biofilm biomass changes due to starvation at each time point was compared to the control grown in $\mathrm{M} 9$ glucose and calculated as $\left(\mathrm{OD}_{550}\right.$ starved $-\mathrm{OD}_{550}$ control) $/ \mathrm{OD}_{550}$ control $\times 100 \%$. The percentage biofilm changes were plotted against time and the rate of change of biofilm reduction was obtained by determining the slope of the line. Data were obtained from three independent replicates with two technical replicates each. ANOVA analysis was carried out using GraphPad Prism v8.1.0 followed by Tukey's post-test.

\section{Abbreviations}

RSCV: rugose small colony variants; SCV: small colony variants; WT: wild type; c-di-GMP: cyclic diguanylate; SNP: single nucleotide polymorphism; SNV: single nucleotide variant.

\section{Acknowledgements}

We would like to thank Tim Tolker-Nielsen for providing the c-di-GMP reporter plasmid pCdrA::gfp (ASV). We would like to thank sequencing facility at SCELSE, NTU for genome sequencing.

\section{Authors' contributions}

SAR, DM and SK designed the experiments. HASN, SS, WHP, NTBH and MT performed the experiments. HASN, SS, DM and SAR wrote the manuscript. All other authors have contributed to data collection and interpretation, and critically reviewed the manuscript. All authors read and approved the final manuscript.

\section{Funding}

The authors would like to acknowledge the financial support from National Research Foundation and Ministry of Education Singapore under its Research Centre of Excellence Program, a grant from the Singapore Ministry of Education (MOE2019-T2-1-050) and Nanyang Technological University for research scholarship for HASN. Funding was also provided through the Australian Research Council (DP140102192).

\section{Availability of data and materials}

All data generated or analyzed during this study are included in this published article. The genome sequence data supporting the conclusions of this article are available in the NCBI SRA database with BioProject accession number PRJNA733671 (http://www.ncbi.nlm.nih.gov/bioproject/733671).

\section{Declarations}

Ethics approval and consent to participate

Not applicable.

\section{Consent for publication}

Not applicable.

\section{Competing interests}

The authors declare they have no competing interests.

\section{Author details}

${ }^{1}$ The Singapore Centre for Environmental Life Sciences Engineering, Singapore, Singapore. ${ }^{2}$ Interdisciplinary Graduate School, Singapore, Singapore. ${ }^{3}$ Present address: Eppendorf AG, Barkhausenweg 1, 22339 Hamburg, Germany. ${ }^{4}$ Present address: Public Utilities Board, Government of Singapore, Singapore, Singapore. ${ }^{5}$ Costerton Biofilm Center, Faculty of Health and Medical Sciences, University of Copenhagen, Copenhagen, Denmark. ${ }^{6}$ School of Biological Sciences, Nanyang Technological University, Singapore, Singapore. ${ }^{7}$ The Ithree Institute, University of Technology Sydney, Sydney, Australia.

Received: 18 May 2021 Accepted: 14 September 2021

Published online: 22 September 2021

\section{References}

1. Harmsen M, Yang L, Pamp SJ, Tolker-Nielsen T. An update on Pseudomonas aeruginosa biofilm formation, tolerance, and dispersal. FEMS Immunol Med Microbiol. 2010;59(3):253-68. https://doi.org/10.1111/j. 1574-695X.2010.00690.x.

2. Gjermansen M, Ragas P, Sternberg C, Molin S, Tolker-Nielsen T. Characterization of starvation-induced dispersion in pseudomonas putida biofilms. Environ Microbiol. 2005;7(6):894-904.

3. Barraud N, Hassett DJ, Hwang S-H, Rice SA, Kjelleberg S, Webb JS. Involvement of nitric oxide in biofilm dispersal of Pseudomonas aeruginosa. J Bacteriol. 2006;188(21):7344-53.

4. Schleheck D, Barraud N, Klebensberger J, Webb JS, McDougald D, Rice $\mathrm{SA}$, et al. Pseudomonas aeruginosa PAO1 preferentially grows as aggregates in liquid batch cultures and disperses upon starvation. PLoS One. 2009;4(5)e5513.

5. Morgan R, Kohn S, Hwang S-H, Hassett DJ, Sauer K. BdlA, a Chemotaxis regulator essential for biofilm dispersion in Pseudomonas aeruginosa. J Bacteriol. 2006;188(21):7335-43. 
6. McDougald D, Rice SA, Barraud N, Steinberg PD, Kjelleberg S. Should we stay or should we go: mechanisms and ecological consequences for bacterial dispersal from biofilms. Nat Rev Microbiol. 2012;10:39-50.

7. Reynolds MM, Frost MC, Meyerhoff ME. Nitric oxide-releasing hydrophobic polymers: preparation, characterization, and potential biomedical applications. Free Radic Biol Med. 2004;37(7):926-36.

8. Barraud N, Storey MV, Moore ZP, Webb JS, Rice SA, Kjelleberg S. Nitric oxide-mediated dispersal in single-and multi-species biofilms of clinically and industrially relevant microorganisms. Microb Biotechnol. 2009;2(3):370-8.

9. Barraud N, Schleheck D, Klebensberger J, Webb JS, Hassett DJ, Rice SA, et al. Nitric oxide signaling in Pseudomonas aeruginosa biofilms mediates phosphodiesterase activity, decreased cyclic di-GMP levels, and enhanced dispersal. J Bacteriol. 2009;191(23):7333-42. https://doi.org/ 10.1128/jb.00975-09.

10. Huynh TT, McDougald D, Klebensberger J, Al Qarni B, Barraud N, Rice SA, et al. Glucose starvation-induced dispersal of Pseudomonas aeruginosa biofilms is CAMP and energy dependent. PLoS One. 2012;7(8):e42874. https://doi.org/10.1371/journal.pone.0042874.

11. Barnes RJ, Bandi RR, Seng WW, Barraud N, McDougald D, Fane AG, et al. Optimal dosing regimen of nitric oxide donor compounds for the reduction of Pseudomonas aeruginosa biofilm and the applicability to wastewater membrane bacterial isolates. Biofouling. 2013;29(2):203-12

12. Xu KD, Stewart PS, Xia F, Huang C-T, McFeters GA. Spatial physiological heterogeneity in Pseudomonas aeruginosa biofilm is determined by oxygen availability. Appl Environ Microbiol. 1998;64(10):4035-9.

13. Kühl M, Rickelt LF, Thar R. Combined imaging of bacteria and oxygen in biofilms. Appl Environ Microbiol. 2007;73(19):6289-95.

14. De Beer D, Stoodley P, Roe F, Lewandowski Z. Effects of biofilm structures on oxygen distribution and mass transport. Biotechnol Bioeng. 1994:43(11):1131-8.

15. Stewart PS, Franklin MJ. Physiological heterogeneity in biofilms. Nat Rev Microbiol. 2008;6(3):199-210.

16. Nair HA. C-di-GMP distribution across single and mixed species biofilms. PhD Thesis. 2016.

17. Kirov SM, Webb JS, Che Y, Reid DW, Woo JK, Rice SA, et al. Biofilm differentiation and dispersal in mucoid Pseudomonas aeruginosa isolates from patients with cystic fibrosis. Microbiology. 2007;153(10):3264-74

18. Koh KS, Lam KW, Alhede M, Queck SY, Labbate M, Kjelleberg S, et al. Phenotypic diversification and adaptation of Serratia marcescens MG1 biofilm-derived morphotypes. J Bacteriol. 2007;189(1):119-30.

19. Irie Y, Borlee BR, O'Connor JR, Hill PJ, Harwood CS, Wozniak DJ, et al. Self-produced exopolysaccharide is a signal that stimulates biofilm formation in Pseudomonas aeruginosa. Proc Natl Acad Sci U S A. 2012;109(50):20632-6.

20. McElroy KE, Hui JG, Woo JK, Luk AW, Webb JS, Kjelleberg S, et al. Strainspecific parallel evolution drives short-term diversification during Pseudomonas aeruginosa biofilm formation. Proc Natl Acad Sci U S A 2014;111(14):E1419-27. https://doi.org/10.1073/pnas.1314340111.

21. Mai-Prochnow A, Evans F, Dalisay-Saludes D, Stelzer S, Egan S, James $S$, et al. Biofilm development and cell death in the marine bacterium Pseudoalteromonas tunicata. Appl Environ Microbiol. 2004;70(6):32328. https://doi.org/10.1128/AEM.70.6.3232-3238.2004.

22. Lee KWK, Yam KHJ, Periasamy S, Mukherjee M, Kjelleberg S, Rice SA. Interspecific diversity reduces and functionally substitutes for intraspecific variation in biofilm communities. ISME J. 2016;10:846-57. https:// doi.org/10.1038/ismej.2015.159.

23. Lee KW, Periasamy S, Mukherjee M, Xie C, Kjelleberg S, Rice SA. Biofilm development and enhanced stress resistance of a model, mixedspecies community biofilm. ISME J. 2014;8(4):894-907. https://doi.org/ 10.1038/ismej.2013.194.

24. Kirov SM, Webb JS, O'May CY, Reid DW, Woo JKK, Rice SA, et al. Biofilm differentiation and dispersal in mucoid Pseudomonas aeruginosa isolates from patients with cystic fibrosis. Microbiol. 2007:153(10):3264-74.

25. Malone JG, Williams R, Christen M, Jenal U, Spiers AJ, Rainey PB. The structure-function relationship of WspR, a Pseudomonas fluorescens response regulator with a GGDEF output domain. Microbiol. 2007;153:980-94.

26. D'Argenio DA, Calfee MW, Rainey PB, Pesci EC. Autolysis and autoaggregation in Pseudomonas aeruginosa colony morphology mutants. J Bacteriol. 2002;184(23):6481-9.

27. Comolli JC, Hauser AR, Waite L, Whitchurch CB, Mattick JS, Engel JN. Pseudomonas aeruginosa gene products PilT and PilU are required for cytotoxicity in vitro and virulence in a mouse model of acute pneumonia. Infect Immun. 1999;67(7):3625-30.

28. Misic AM, Satyshur KA, Forest KT. P. aeruginosa PilT structures with and without nucleotide reveal a dynamic type IV pilus retraction motor. J Mol Biol. 2010;400(5):1011-21. https://doi.org/10.1016/j.jmb. 2010.05.066.

29. Mougous JD, Gifford CA, Ramsdell TL, Mekalanos JJ. Threonine phosphorylation post-translationally regulates protein secretion in Pseudomonas aeruginosa. Nat Cell Biol. 2007;9(7):797-803. https://doi. org/10.1038/ncb1605.

30. Hickman JW, Tifrea DF, Harwood CS. A chemosensory system that regulates biofilm formation through modulation of cyclic diguanylate levels. Proc Natl Acad Sci U S A. 2005;102(40):14422-7.

31. Bantinaki E, Kassen R, Knight CG, Robinson Z, Spiers AJ, Rainey PB. Adaptive divergence in experimental populations of Pseudomonas fluorescens. III. Mutational origins of wrinkly spreader diversity. Genetics. 2007;176(1):441-53. https://doi.org/10.1534/genetics.106.069906.

32. Fleiszig SM, Arora SK, Van R, Ramphal R. FlhA, a component of the flagellum assembly apparatus of Pseudomonas aeruginosa, plays a role in internalization by corneal epithelial cells. Infect Immun. 2001;69(8):4931-7. https://doi.org/10.1128/IAl.69.8.4931-4937.2001.

33. Starkey M, Hickman JH, Ma L, Zhang N, De Long S, Hinz A, et al. Pseudomonas aeruginosa rugose small-colony variants have adaptations that likely promote persistence in the cystic fibrosis lung. J Bacteriol. 2009;191(11):3492-503.

34. Chua SL, Liu Y, Yam JKH, Chen Y, Vejborg RM, Tan BGC, et al. Dispersed cells represent a distinct stage in the transition from bacterial biofilm to planktonic lifestyles. Nat Commun. 2014;5. https://doi.org/10.1038/ ncomms5462.

35. Zhu X, Oh HS, Ng YCB, Tang PYP, Barraud N, Rice SA. Nitric OxideMediated Induction of Dispersal in Pseudomonas aeruginosa Biofilms Is Inhibited by Flavohemoglobin Production and Is Enhanced by Imidazole. Antimicrobial Agents Chemotherapy. 2018;62(3); doi: https://doi. org/10.1128/AAC.01832-17.

36. Boles BR, Thoendel M, Singh PK. Self-generated diversity produces "insurance effects" in biofilm communities. Proc Natl Acad Sci. 2004;101(47):16630-5.

37. Minamino T, Macnab RM. FliH, a soluble component of the type III flagellar export apparatus of Salmonella, forms a complex with Flil and inhibits its ATPase activity. Mol Microbiol. 2000;37(6):1494-503.

38. Tian Z-X, Mac Aogain M, O'Connor HF, Fargier E, Mooij MJ, Adams C, et al. MexT modulates virulence determinants in Pseudomonas aeruginosa independent of the MexEF-OprN efflux pump. Microb Pathog. 2009:47(4):237-41.

39. Nair HA, Periasamy S, Yang L, Kjelleberg S, Rice SA. Real time, spatial, and temporal mapping of the distribution of c-di-GMP during biofilm development. J Biol Chem. 2017;292(2):477-87. https://doi.org/10. 1074/jbc.M116.746743.

40. Tolker-Nielsen T, Sternberg C. Methods for studying biofilm formation: flow cells and confocal laser scanning microscopy. Methods Molecular Biol. 2014;1149:615-29; doi: https://doi.org/10.1007/978-14939-0473-0_47.

41. Abràmoff MD, Magalhães PJ, Ram SJ. Image processing with ImageJ. Biophoton Int. 2004;11(7):36-42.

42. Lee KKW. Role of cell-cell signaling, differentiation and fitness in multispecies biofilms. PhD Thesis. 2014.

\section{Publisher's Note}

Springer Nature remains neutral with regard to jurisdictional claims in published maps and institutional affiliations. 